\title{
Clinical Outcomes of Negative-Pressure Wound Therapy with Instillation without Commercial Devices in the Treatment of Complex Wounds
}

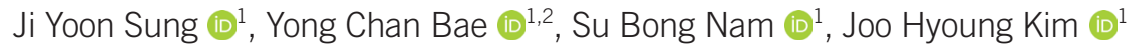 \\ ${ }^{1}$ Department of Plastic and Reconstructive Surgery, Pusan National University School of Medicine, Busan; ${ }^{2}$ Biomedical Research Institute, Pusan National \\ University Hospital, Busan, Korea
}

\begin{abstract}
Background: Negative-pressure wound therapy with instillation (NPWTi) is an adjunctive treatment modality for complex and infected wounds. However, commercial devices are expensive and not readily available in many countries. The objective of this study is to introduce an NPWTi method that is applicable where commercial NPWTi devices are not available and to report the clinical outcomes of the NPWTi method for the adjunctive treatment of complex wounds.

Methods: This prospective clinical experimental study included 51 patients who had wounds on which operative debridement was performed between January 2017 and March 2019. A negative-pressure wound therapy (NPWT) device was applied with an intravenous (IV) line for continuous instillation of $0.9 \%$ normal saline plus $1 \%$ povidone-iodine solution for chronic wounds. The outcomes measured were the number of operating room visits, time to final surgical procedure, number of infected wounds, time to resolution of infection, type of reconstruction operation, and occurrence of complications.

Results: The average number of operations performed was $2.5 \pm 0.8$, and the time to final surgical procedure was $28.4 \pm 15.4$ days. The number of infected wounds was $35(68.6 \%)$, and the time to resolution of infection was $15.0 \pm 14.6$ days. All wounds were closed or covered. Though partial graft failure occurred in two cases, they healed completely by secondary healing in 2 weeks.

Conclusion: A continuous-instillation NPWT system using an IV line could be an adjunctive modality in treating complex wounds at institutions where commercial NPWTi systems are not readily available.
\end{abstract}

Keywords: Negative-pressure wound therapy; Wound healing; Wounds

Original Article

\section{Introduction}

Negative-pressure wound therapy with instillation (NPWTi) has recently become an adjunctive treatment modality for complex and infected wounds. It is applied over the wound surface in a manner similar to standard negative-pressure wound therapy (NPWT) but with the additional component of instilling antibiotic and/or antiseptic fluid via a second tube system. In 1998, Fleischmann et al. [1] described this concept using a polyvinyl alcohol sponge, drainage tubes, and vacuum pump as an adjunct therapy for a variety of acutely and chronically infected wounds.

The development of automated, controlled, commercially available systems has enabled the delivery of continuous or intermittent controlled volumes of solution to wounded tissues. However, commercial NPWTi devices are not available in many countries, and are more expensive than traditional NPWT systems.

The objective of this study is to introduce an NPWTi method that is applicable where commercial NPWTi devices are not readily available, and to report the clinical outcomes of the NPWTi method for the adjunctive treatment of complex wounds.
Received: July 23, 2019

Revised: October 23, 2019

Accepted: October 24, 2019

\section{Corresponding author:}

Joo Hyoung Kim, M.D., Ph.D.

Department of Plastic and Reconstructive Surgery, Pusan National University School of Medicine, 179 Gudeok-ro, Seo-gu, Busan 49241, Korea

Tel: +82-51-240-7269

Fax: +82-51-243-9405

E-mail: medic144@hanmail.net

This article was presented at PRS Korea 2018 on November 9-11, 2018, in Seoul, Korea and the Wound Meeting Seoul 2019 on March 2829, 2019, in Seoul, Korea.

This is an Open Access article distributed under the terms of the Creative Commons Attribution Non-Commercial License (https://creativecommons.org/licenses/by-nc/4.0/) which permits unrestricted non-commercial use, distribution, and reproduction in any medium, provided the original work is properly cited.

C 2020 Korean Wound Management Society 


\section{Methods}

This single-institution, prospective clinical experimental study enrolled patients who were admitted to a tertiary referral hospital with complex wounds and required surgical debridement in the operating room between January 2017 and March 2019. We defined "complex wounds" using a previously proposed definition in a review article [2], and included patients with wounds complicated by invasive infection or extensive biofilm, massive soft tissue infections (necrotizing fasciitis, Fournier's

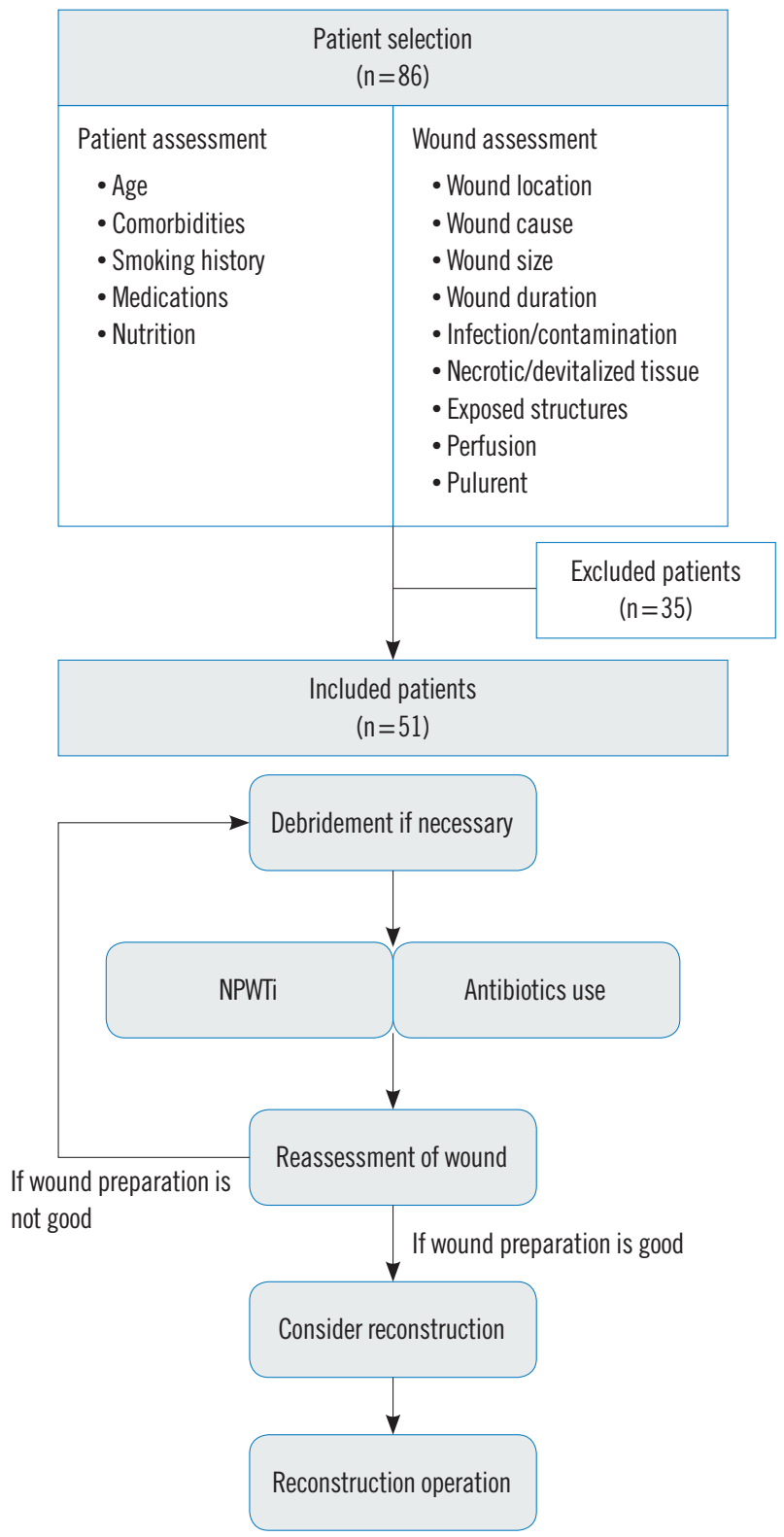

Fig. 1. Study protocol of wounds with NPWTi. NPWTi, negativepressure wound therapy with instillation. gangrene, and gas gangrene), wounds in which healing progression had "stalled" following traditional NPWT therapy, dehisced/infected surgical wounds that have not healed using traditional NPWT therapy, severe traumatic wounds with exposed critical structures (bone, tendon, and muscle), inflammation with exposed surgical materials or implants, and/or diabetic foot wound infections. Patients whose general condition or situation was not eligible for reconstructive surgery or patients who refused reconstructive surgery were excluded from the study (Fig. 1). The treatment modalities performed in this study were accepted by the patients, and they gave written informed consent. The study was approved by the Institutional Review Board of Pusan National University Medical Center (IRB No. H-1907-021-081) and was performed in accordance with the principles of the Helsinki Declaration of 1975 (revised 2008).

The study protocol is shown in Fig. 1. All patients were excisionally debrided by a single surgeon (JHK) using sharp technique in the operating room. Devitalized and infected tissues were removed, tunnels and abscess pockets were explored, and the capsules surrounding the tunnels and pockets were excised. An absorbable collagen hemostat (Novacol Fibrillar; Kyeron, Enschede, the Netherlands) was applied for hemostasis. Tulle-gras dressing (Bactigras; Smith \& Nephew, London, UK) was applied on the wound surface to block the adherence of reticulated open-pore polyurethane foam of the NPWT dressing. An NPWT system (CuraVac; CGbio, Seongnam, Korea) was placed over it with an intravenous (IV) line placed in the deepest surface of the wound to allow for instillation of the antiseptic solution (Fig. 2). A $0.9 \%$ normal saline plus $1 \%$ povidone-iodine solution infiltrated through the IV line continuously. An instillation solution was made using a 3 L, 0.9\% normal saline bag, after removing $300 \mathrm{~mL}$ of normal saline

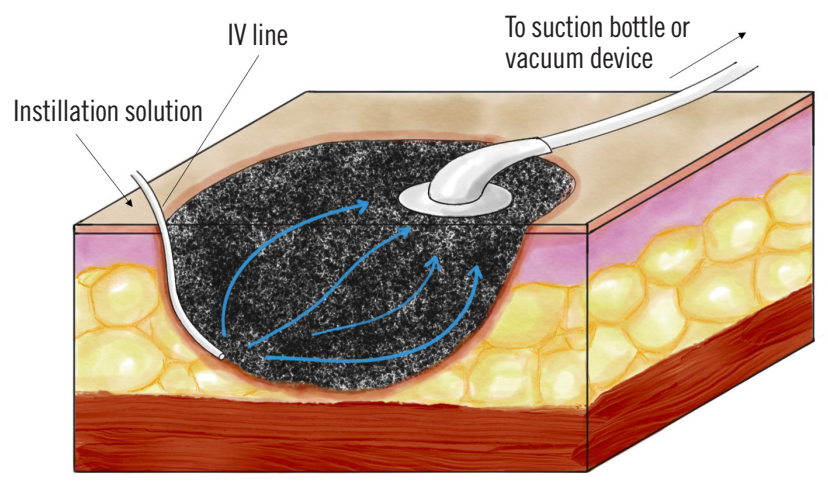

Fig. 2. Schema of NPWTi application with IV line. NPWTi, negative-pressure wound therapy with instillation; IV, intravenous. 
from the bag and adding $300 \mathrm{~mL}$ of $10 \%$ povidone-iodine solution. Three liters of instillation solution was instilled daily.

The mode of negative pressure was continuous, with pressure set at $125 \mathrm{mmHg}$ by the suction drainage system in the ward (Fig. 3). Dressing changes were performed routinely every 5 to 7 days depending on clinical impression or equipment failure (leakage). With every change, the wound was reassessed, and wound cultures and photographs were taken.

Patients received IV treatment with appropriate antibiotics after the debridement operation. When additional surgery was needed, the technique performed was determined by the surgical team according to the local conditions of the wound and the patient's general situation. In those cases where the conditions where optimal, closure with local, regional, or free flap coverage with a skin graft was possible. These procedures were accompanied with debridement if necessary (i.e., when there was visible debris or nonviable tissue). We set the end point of the treatment as definitive closure or graft take if wound closure could be reestablished by surgical procedures. After surgical operations, the NPWTi treatment was stopped and dressings were changed to conventional dressings. Study data

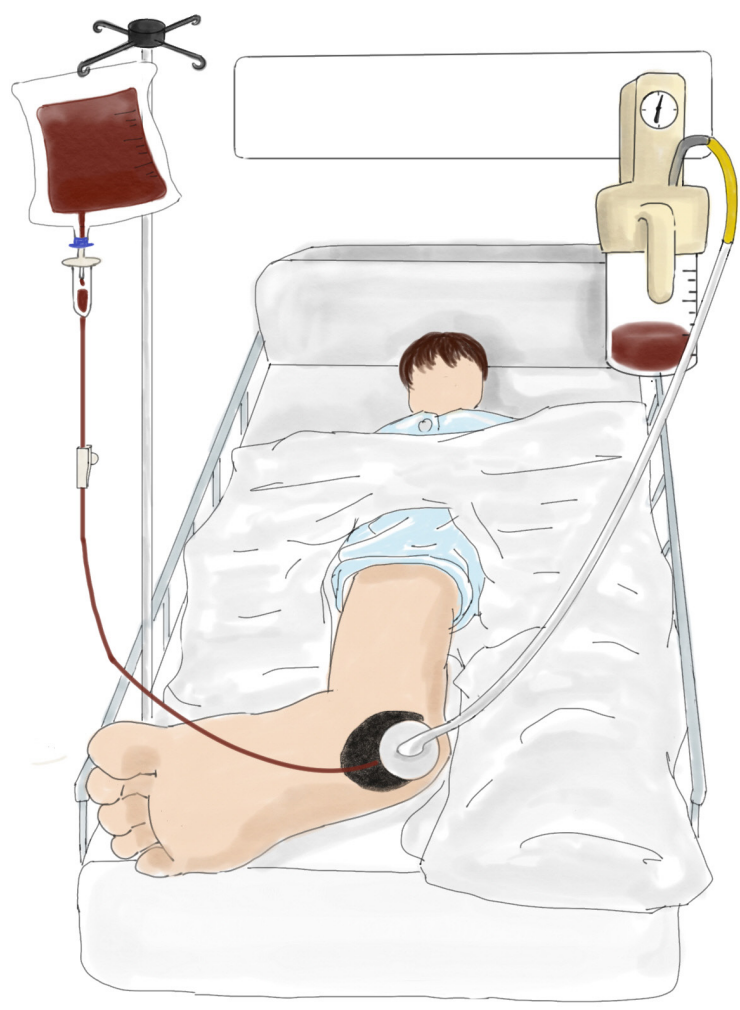

Fig. 3. NPWTi application with the suction drainage system. The application of NPWTi was done in the ward. NPWTi, negativepressure wound therapy with instillation. that were collected included patient demographics, smoking history, comorbidities, wound location, wound cause, proportion of infected wounds, and surrogate wound outcomes.

The surrogate wound outcome was reviewed based on the number of operating room visits, time to final surgical procedure, number of infected wounds, time to resolution of infection, type of reconstruction operation, and occurrence of complications (wound dehiscence, skin graft loss, and recurrence of infection).

\section{Results}

Of the 86 patients who met the inclusion criteria, 35 patients were excluded because their general condition rendered them ineligible for reconstruction operation or because they refused to undergo the reconstruction operation. A total of 51 patients were enrolled in this study. Table 1 shows the patients' demographics, smoking history, and comorbidities.

Wounds were localized mainly at the foot (37.3\%) and lower limbs (excluding the foot) (33.3\%), ischium (13.7\%), trunk (11.8\%), and head and neck (3.9\%) (Table 2). The most frequent cause of wound was surgical (39.2\%), which included wound dehiscence (19.6\%), flap necrosis after free flap operation (17.6\%), and wounds with surgical material (2.0\%). Other causes of wound were trauma, decubitus, neuropathic, necro-

Table 1. Patient demographics, smoking history, and comorbidities $^{\text {a) }}$

\begin{tabular}{lc}
\hline Characteristic & Value $(n=51)$ \\
\hline Sex & \\
Male & $33(64.7)$ \\
Female & $18(35.3)$ \\
Age (yr) & $60.2 \pm 15.1$ \\
Smoking history & $14(27.5)$ \\
Hypertension & $19(37.3)$ \\
Diabetes mellitus & $15(29.4)$ \\
Hyperlipidemia & $4(7.8)$ \\
Cardiovascular disease & $4(7.8)$ \\
End-stage renal disease & $2(3.9)$ \\
Cerebrovascular disease & $2(3.9)$ \\
Peripheral vascular disease & $3(5.9)$ \\
Cancer history & $3(5.9)$ \\
Aortic aneurysm & $1(2.0)$ \\
\hline
\end{tabular}

Values are presented as number (\%) or mean \pm SD.

a)Patients were counted more than once if multiple comorbidities existed. 
Table 2. Summary of wound locations $(n=51)$

\begin{tabular}{lc}
\hline Wound location & No. (\%) \\
\hline Head and neck & $2(3.9)$ \\
Scalp & $1(2.0)$ \\
Neck & $1(2.0)$ \\
Trunk & $6(11.8)$ \\
Back & $5(9.8)$ \\
Abdomen & $1(2.0)$ \\
Ischium & $7(13.7)$ \\
Sacrum & $6(11.8)$ \\
Greater trochanter & $1(2.0)$ \\
Lower limb (except the foot) & $17(33.3)$ \\
Thigh & $5(9.8)$ \\
Lower leg & $7(13.7)$ \\
Ankle & $5(9.8)$ \\
Foot & $19(37.3)$ \\
Foot dorsum & $8(15.7)$ \\
Sole & $4(7.8)$ \\
Heel & $7(13.7)$ \\
\hline
\end{tabular}

tizing fasciitis, inflammation, venous, contact burn, and extravasation (Table 3).

The total number of surgical procedures was 126 , including 75 debridement operations and 51 reconstruction operations, and the average number of operations was $2.5 \pm 0.8$. Time to final surgical procedure, which is same as the NPWTi treatment period, was $28.4 \pm 15.4$ days (Table 4 ). The number of infected wounds was $35(68.6 \%)$ and time to resolution of infection was $15.0 \pm 14.6$ days. All wounds were closed or covered.

The surgical procedures used for reconstruction were local flap (25.5\%), split thickness skin graft (47.1\%), full thickness skin graft (3.9\%), local flap with split thickness skin graft (5.9\%), local flap with full thickness skin graft (2.0\%), superior gluteal artery perforator flap (11.8\%), peroneal artery perforator flap (2.0\%), and anterolateral thigh free flap (2.0\%) (Table 5). There was partial graft failure in two cases, but they were completely healed by secondary healing in 2 weeks (Table 4 ). Wound dehiscence or recurrence of infection was not observed.

\section{Case studies}

The following case studies demonstrate the successful use of NPWTi.
Table 3. Summary of wound causes $(n=51)$

\begin{tabular}{lc}
\hline Wound cause & No. (\%) \\
\hline Surgical & $20(39.2)$ \\
Wound dehiscence & $10(19.6)$ \\
Flap necrosis & $9(17.6)$ \\
Wounds with surgical material or implant & $1(2.0)$ \\
Trauma & $13(25.5)$ \\
Decubitus & $9(17.6)$ \\
Neuropathic & $3(5.9)$ \\
Necrotizing fasciitis & $2(3.9)$ \\
Inflammatory & $1(2.0)$ \\
Venous & $1(2.0)$ \\
Contact burn & $1(2.0)$ \\
Extravasation & $1(2.0)$
\end{tabular}

Table 4. Outcomes of NPWTi $(n=51)$

\begin{tabular}{lc}
\hline Outcome & Value $(n=51)$ \\
\hline No. of operations & $2.5 \pm 0.8$ \\
Time to final surgical procedure (day) & $28.4 \pm 15.4$ \\
No. of infected wounds & $35(68.6)$ \\
Time to resolution of infection (day) & $15.0 \pm 14.6$ \\
Complications after closed/covered & $2(3.9)$ \\
Partial graft failure & $2(3.9)$ \\
Wound dehiscence & 0 \\
Recurrence of infection & 0 \\
\hline
\end{tabular}

Values are presented as mean \pm SD or number (\%).

NPWTi, negative-pressure wound therapy with instillation.

Table 5. Surgical procedures used for reconstruction $(n=51)$

\begin{tabular}{lc}
\hline Surgical procedure & №. (\%) \\
\hline Local flap & $13(25.5)$ \\
Split thickness skin graft & $24(47.1)$ \\
Full thickness skin graft & $2(3.9)$ \\
Local flap with split thickness skin graft & $3(5.9)$ \\
Local flap with full thickness skin graft & $1(2.0)$ \\
Superior gluteal artery perforator flap & $6(11.8)$ \\
Peroneal artery perforator flap & $1(2.0)$ \\
Anterolateral thigh free flap & $1(2.0)$ \\
\hline
\end{tabular}

Case 1

The patient was a 70-year-old man with necrotizing fasciitis and osteomyelitis on the right heel, with exposed bone and tendons. The wound was infected by Corynebacterium and 

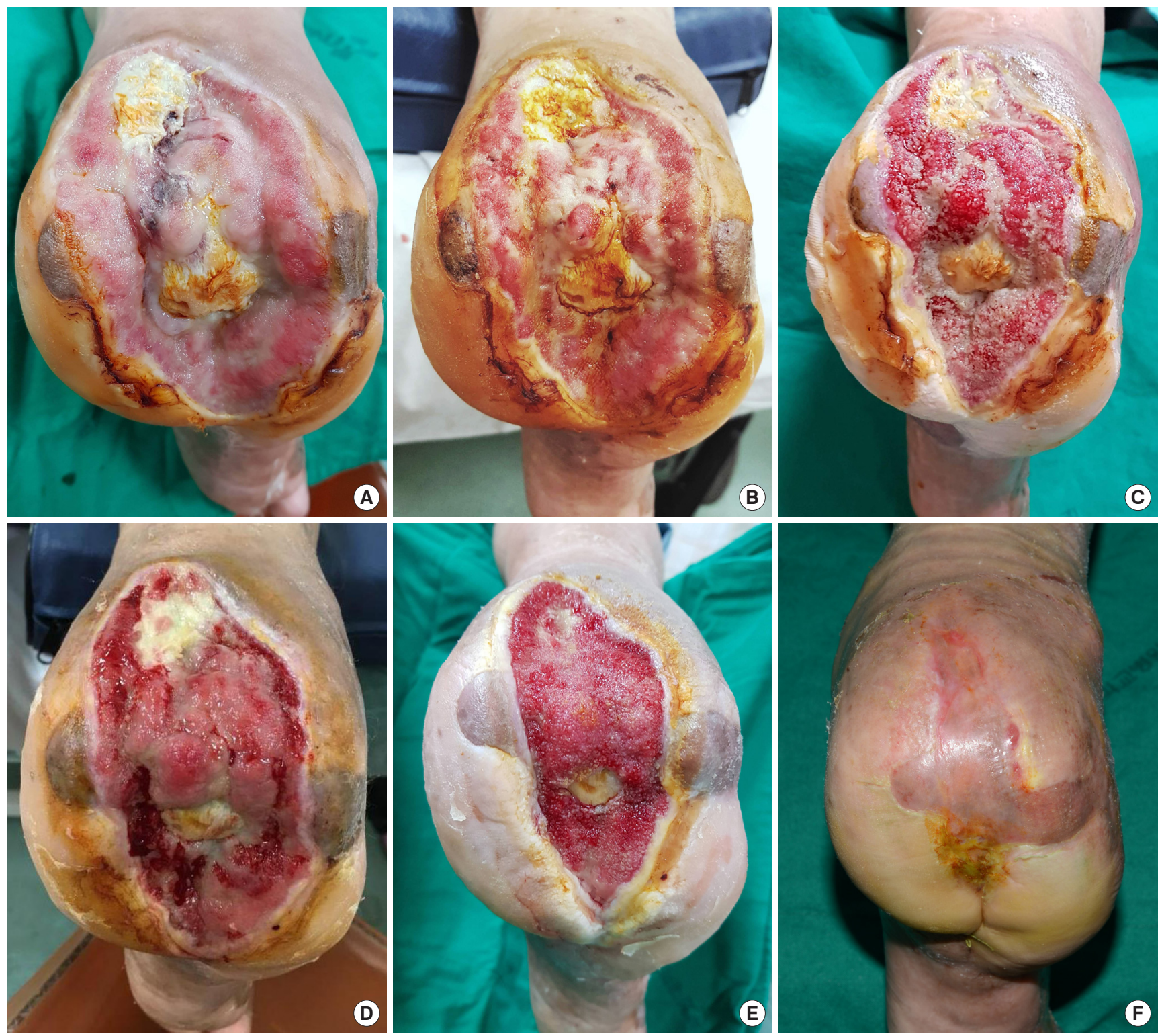

Fig. 4. Necrotizing fasciitis with osteomyelitis on right heel. The right heel before NPWTi (A), after 1 week of NPWTi (B), after 2 weeks of NPWTi (C), after 4 weeks of NPWTi (D), after 7 weeks of NPWTi (E), and at 3 months after split thickness skin graft (F). NPWTi, negative-pressure wound therapy with instillation.

multidrug-resistant Acinetobacter baumannii and had tissue edema. His wound was not healed by conventional NPWT, and a biofilm repeatedly covered the wound surface. After debridement operation, NPWTi was applied to granulate and cleanse the wound and to reduce exudate. We particularly selected instillation solution of hypertonic saline plus povidoneiodine (Betadine) for this patient because of the tissue edema. The wound was treated for 7 weeks with NPWTi. After 7 weeks of NPWTi, infection was resolved, local tissue edema decreased, and the defect size decreased from $12 \times 20$ to $4 \times 16$ $\mathrm{cm}$, with granulation tissue covering the exposed bone and tendon. A split-thickness skin graft was performed, and NPWTi was applied to the split thickness skin graft as adjunctive treatment for 7 days postoperatively. On postoperative days 3 and 5, $1 \mathrm{~L}$ of $0.9 \%$ normal saline plus $1 \%$ povidone-iodine was instilled to prevent infection and dilute the inflammatory exudate. After 3 months, the patient was able to bear weight, ambulatory, and required no dressing (Fig. 4). 

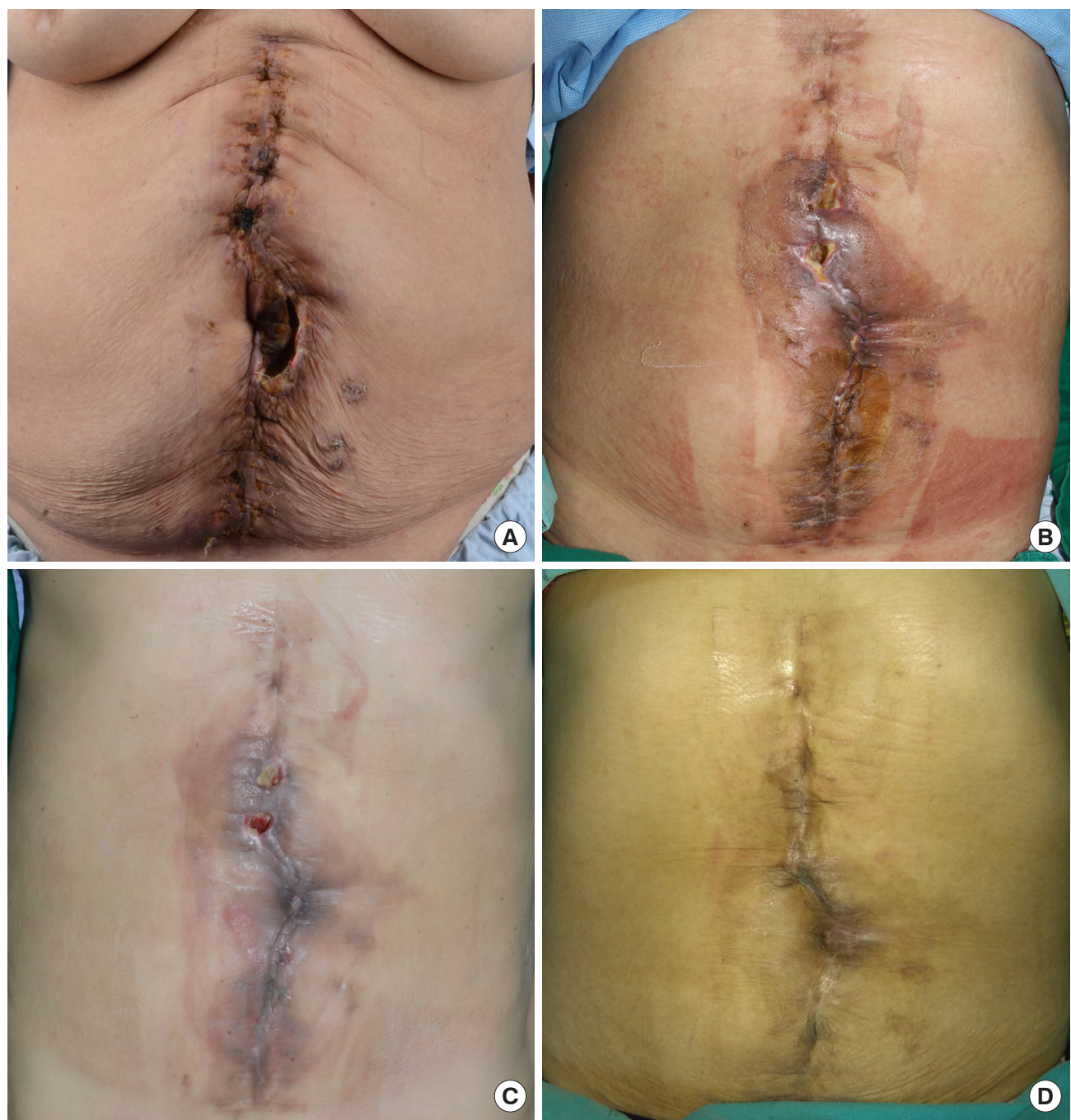

Fig. 5. Wound dehiscence with infection on abdomen. (A) Photograph of before debridement. (B) Wound dehiscence occurred before NPWTi. (C) Photograph of after 11 days of NPWTi. (D) The infection resolved at 1 month after local flap coverage. NPWTi, negativepressure wound therapy with instillation.

\section{Case 2}

The patient was a 75-year-old woman with hypertension and abdominal aortal aneurysm rupture. After the resection of the abdominal aortal aneurysm, wound dehiscence with necrosis and methicillin-resistant Staphylococcus aureus infection occurred on the surgical site. After debridement and local flap coverage, wound dehiscence occurred, with wound tunneling, purulent discharge, and continuous infection. The wound was treated with NPWTi for 4 weeks, and the infection resolved after 11 days. After 4 weeks of NPWTi, closure with local flap operation was performed, and 4 weeks after the operation, the wound had no complications (Fig. 5).

\section{Discussion}

In our study, 51 patients with complex wounds which were not healed with traditional NPWT were treated with a new NPWTi system, and their wounds were all closed or covered with no major complications. NPWTi has the advantage of both standard NPWT and irrigation. Standard NPWT has several advantages in wound healing: wound shrinkage or macrodeformation, microdeformation at the foam-wound surface interface, fluid removal, stabilization of the wound environment, and the secondary effects of wound healing including angiogenesis, neurogenesis, granulation tissue formation, and cellular proliferation, differentiation and migration [3].

Studies comparing NPWTi with standard treatment or 
NPWT showed statistically significant difference in infection recurrence [4], length of hospital stay [4-6], time for wound healing [6,7], absolute reduction in bacterial load in the infection bed [8], and bedside efficacy and safety [6]. Clinical indications for NPWTi include osteomyelitis, diabetic foot syndrome, necrotizing fasciitis, chronic infections of surgical implants, and NPWTi can be used as a prophylactic procedure for contaminated traumatic wounds as well $[4,9,10]$.

The development of automated, controlled, commercially available NPWTi systems has enabled the delivery of continuous or intermittent controlled volumes of solution to wounded tissues. However, commercial NPWTi systems are not always readily available in many countries, and are more expensive than traditional NPWT systems. Therefore, we designed a standardized NPWTi system with a traditional NPWT system, an IV line, and a vacuum system that can be easily devised and used in hospitals where commercial NPWTi systems are not available. Our continuous-instillation NPWT system can be a variation of NPWTi with a suction port and an IV line-based continuous-drip system.

There are few studies utilizing preexisting instruments and NPWT to work as NPWTi systems. Kiyokawa et al. [11] introduced NPWT with continuous irrigation system placing two thick tubes with holes inside the wound. A total of 17 cases with nine closed wound cases and eight open wound cases were treated with this system, and granulation of the wound surface improved dramatically and the infection was almost eliminated. Sakakibara et al. [12] introduced NPWT with focal irrigation utilizing a drain tube. In 11 out of 12 cases, infection was controlled and wound bed preparation was achieved.

Our system has several advantages compared with commercial NPWTi systems. First, it is cost-effective compared with commercial NPWTi systems. The specific costs of commercial NPWTi are determined by consumables such as the instillation bag unit (V.A.C. VeraLink Cassette, KCI Clinic Spain SL, Pozuelo de Alarcón, Spain; one-time cost of \$55), NPWT dressing (V.A.C. Veraflow dressing, KCI Clinic Spain SL; $\$ 63.25$ per dressing), and canister ( $\$ 42.49$ per dressing) [6]. For our NPWTi system, no instillation bag unit or canister is needed. Second, because we use the suction drainage in the ward, there is no limit to the amount of the instillation solution that can be used in our NPWTi system. Whereas for commercial NPWTi systems, canisters are single-use and have specific volumes $(300,500$, or $1,000 \mathrm{~mL})$; the volume of the instillation solution is limited by the canister's volume. Third, the advantage of using an IV line for instillation system is that it can be placed in deep wounds, tunnels, or even in fistula. It is worth noting that in commercial NPWTi systems, the instillation system with vacuum is placed on top of the dressing, potentially failing to bring the solution deep into the wound, only providing a superficial instillation and making a shunt from the instillation tube directly to the vacuum tube. Solution dwell times were thus conceived for commercial NPWTi as a means to address the shunting. In our system, the shunting issue is solved by placing the IV line deep in the wound surface, taking into account which way the solution will flow.

Meanwhile, there are also several disadvantages in our system. First, patients have to be admitted to the hospital to undergo this treatment because the instillation solution needs to be changed daily and the suction drainage system is in the ward. Second, dwell time has to be set manually. Third, while the commercial NPWTi can keep constant negative pressure on the wound surface, our NPWTi system will not provide constant pressure if patients change their position.

As no consensus or guidelines have been established on the instillation of antibiotics or on the stability of antibiotic or antiseptic solutions [13], it is possible to instill normal saline or other agents (i.e., antibiotics and antiseptics). Kim et al. [5] showed no statistically significant difference between $0.9 \%$ normal saline and an antiseptic ( $0.1 \%$ polyhexanide plus $0.1 \%$ betaine) for NPWTi in a prospective, randomized, comparative effectiveness study. Based on our clinical experience, we routinely use $0.9 \%$ normal saline plus $1 \%$ povidone-iodine solution.

There are several limitations to our study. First, the sample size is small, which is typical for studies associated with new treatments. Second, there is no control group. Statistical comparison between NPWTi and conventional dressing or NPWT was not conducted because we selected patients who had complex wounds that were not healed by conventional dressings. The selected patients who were not healed by conventional dressings were closed or covered completely after NPWTi. Further case-control studies with larger samples are needed in the future to clarify the effectiveness of this system.

Our study is the first clinical experimental study in Korea conducted in standardized fashion utilizing an NPWT system without commercial NPWTi systems. A variety of instillation solutions can be selected depending on local conditions of the wound. In addition, the volume and velocity of the instillation solution can be controlled by adjusting the tube clamping device on the IV line, or with more precision with an infusion pump device. The negative pressure can also be adjusted ac- 
cording to conditions of wound. In this study, the negative pressure was set at $125 \mathrm{mmHg}$, which was in accordance with consensus guidelines published in 2015 [2]. This new NPWTi system can be used easily at institutions where commercial NPWTi systems are not readily available, and it is also more cost-effective than commercial NPWTi systems. Therefore, this NPWTi method will provide a standardized and inexpensive system in treating complex wounds. In conclusion, a continuous-instillation NPWT system with a suction port and IV line functioning as a continuous-drip system could be an adjunctive modality in treating complex wounds, making it a practical option in wound management.

\section{Conflict of interest}

No potential conflicts of interest relevant to this article are reported.

\section{ORCID iDs}

$\begin{array}{ll}\text { Ji Yoon Sung } & \text { https://orcid.org/0000-0001-6925-8120 } \\ \text { Yong Chan Bae } & \text { https://orcid.org/0000-0002-0268-4667 } \\ \text { Su Bong Nam } & \text { https://orcid.org/0000-0002-9661-0879 } \\ \text { Joo Hyoung Kim } & \text { https://orcid.org/0000-0002-4893-3761 }\end{array}$

\section{References}

1. Fleischmann W, Russ M, Westhauser A, et al. Vacuum sealing as carrier system for controlled local drug administration in wound infection. Unfallchirurg 1998;101:649-54.

2. Kim PJ, Attinger CE, Crist BD, et al. Negative pressure wound therapy with instillation: review of evidence and recommendations. Wounds 2015;27:S2-S19.

3. Huang C, Leavitt T, Bayer LR, et al. Effect of negative pressure wound therapy on wound healing. Curr Probl Surg 2014;51:301-31.

4. Timmers MS, Graafland N, Bernards AT, et al. Negative pressure wound treatment with polyvinyl alcohol foam and polyhexanide antiseptic solution instillation in posttraumatic osteomyelitis. Wound Repair Regen 2009;17:
278-86.

5. Kim PJ, Attinger CE, Oliver N, et al. Comparison of outcomes for normal saline and an antiseptic solution for negative-pressure wound therapy with instillation. Plast Reconstr Surg 2015;136:657e-664e.

6. Gabriel A, Kahn K, Karmy-Jones R. Use of negative pressure wound therapy with automated, volumetric instillation for the treatment of extremity and trunk wounds: clinical outcomes and potential cost-effectiveness. Eplasty 2014;14:e41.

7. Gabriel A, Shores J, Heinrich C, et al. Negative pressure wound therapy with instillation: a pilot study describing a new method for treating infected wounds. Int Wound J 2008;5:399-413.

8. Goss SG, Schwartz JA, Facchin F, et al. Negative pressure wound therapy with instillation (NPWTi) better reduces post-debridement bioburden in chronically infected lower extremity wounds than NPWT alone. J Am Coll Clin Wound Spec 2014;4:74-80.

9. Lehner B, Fleischmann W, Becker R, et al. First experiences with negative pressure wound therapy and instillation in the treatment of infected orthopaedic implants: a clinical observational study. Int Orthop 2011;35:1415-20.

10. Back DA, Scheuermann-Poley C, Willy C. Recommendations on negative pressure wound therapy with instillation and antimicrobial solutions - when, where and how to use: what does the evidence show? Int Wound J 2013;10 Suppl 1:32-42.

11. Kiyokawa K, Takahashi N, Rikimaru H, et al. New continuous negative-pressure and irrigation treatment for infected wounds and intractable ulcers. Plast Reconstr Surg 2007; 120:1257-65.

12. Sakakibara S, Osawa S, Kitani K, et al. Negative pressure wound therapy with focal irrigation using a preexisting instrument. Sosyo 2016;7:110-7.

13. Cortell-Fuster C, Gaspar-Carreno M, Achau-Munoz R, et al. Negative pressure therapy with instillation for the treatment of infected wounds: recommendations of utilization based on evidence. Farm Hosp 2019;43:6-12. 